\title{
The Scalar Form of Jacobi's Equations in the Calculus of Variations.
}

\author{
Memoria di Hanno Rund (a Bonn - Germany).
}

Snmmary. - The tensorial equations of geodesic deviation in a generalised metric (FINSLER) space of $n$ dimensions are reduced to a single equation in scalar form involving only the length of the infinitesimal variation vector. The terms occurring in this equation are interpreted geometrically by means of the theory of subspaces. An expression for the second variation of the length integral is determined, from which the differential equations of the accessory problems are obtained. These, the so-called $J_{\mathrm{ACOBI}}$ equations, are shown to contain the above-mentioned scalar form of the equation of geadesic deviation as a special case. This leads to a novel general discussion of the accessory problem in Calculus of Variations.

\section{\$ 1. - The Equation of Geodesic Deviation.}

In a recent paper the present author $[1]\left(^{1}\right)$ derived the equation of geodesic deviation in a FrNSLER space as a generalisation of the corresponding equations for the case of a Riemannian space due to Levi-Civita [2]. In order to preserve the geometrical aspect of the theory the notion of element of support, which has become customary in the theory of FinsLer spaces, was strictly avoided; and hence the author's [3] covariant derivative, which has recently been obtained once more from a different point of view by E. Bomplani [4], was used throughout and will furthermore play an essential rôle in the present paper. In [1] a scalar form of the equations of geodesic deviation (i. e. a single equation involving the length of the variation vector) for the case of a two-dimensional FinsLer space had been obtained. The purpose of the present paper is partly to derive this form of the equations of geodesic deviation for the general n-dimensional case, and to interpret these results geometrically in the light of recent results obtained in the theory of subspaces of a FINsLen space. This will enable us to establish the natural generalisations of results due to ONIOESCU [5] and BoRTOLOTII [6] for the case of a Riemannian space. In $\S 2$ we shall then derive an expression for the second variation of the length integral in FunsLen spaces, by means of which we can generalise a number of theorems valid in Riemannian spaces as developed by SYNGE [7]. It will be shown in $\$ 3$ that the abovementioned form of the equations of geodesic deviation are a special case of

(1) Numbers in square brackets refer to the bibliography at the end of this paper. 
the $J_{A C O B I}$ equations of the original problem in the Caleulus of Variations defining our Frnster space. This leads to a geometrical theory of the accessory problem as well as to new interpretations of the curvature tensor. In a later paper these results will be applied to the problem of stability of general (relativistic) dynamical systems.

We consider two geodesics $E_{1}$ and $E_{2}$ issuing from the same point $O$ of our Finster space $F_{n}$, and we suppose that $E_{1}$ and $E_{2}$ are neighbouring geodesics in the sense that their tangent vectors at $O$ form an infinitesimal angle $d \varphi$ (as defined in [8], $\S 2$ ) in the Minkowskian tangent space $T_{n}(0)$ at $O$. Let us denote by $x^{i}(s)$ the coordinates of a point $P$ of $E_{1}$ distant $s$ from $O$ (measured along $E_{1}$ ) and by $\psi^{i}(s)$ the coordinates of a point $Q$ of $E_{2}$, also distant $s$ from $O$ (measured along $E_{2}$ ). By writing

$$
\xi^{i}=\psi^{i}(s)-x^{i}(s), \quad(i, j, \ldots=1, \ldots, n),
$$

we can regard $\xi^{\prime}$ (up to quantities of third order) as an infinitesimal vector in $T_{n}(P)$, viz. as the infinitesimal variation vector. It can be shown that under these circumstances (Cf. [1], p. 9) $\xi^{\prime}$ is normal with respect to the tangent vector $x^{\prime i}$ of $E_{1}$ at $P$. Analytically this means that we have $y_{t} \xi^{i}=0$, where $y_{i}=g_{i j}\left(x, x^{\prime}\right) x^{\prime j}, x^{\prime j}=d x^{i} / d s$. In passing we note that this notion of normality is not in general symmetrical, (Cf. [8], \& 2), the metric tensor of $F_{n}$ being defined as usual. Furthermore, $D \xi^{i} / D s$ (here the definition of covariant derivative corresponds to that defined in [3]) is also normal with respect to $x^{i}$ (Cf. [1], p. 6). Let $X^{i}$ be the vector in the direction of $\xi^{i}$, for which we stipulate

$$
\xi X^{i}=\xi^{i}, \quad \text { where } \quad g_{i j}\left(x, x^{\prime}\right) X^{i} X^{j}=1,
$$

and on differentiating this equation covariantly we find

$$
\frac{D \xi^{i}}{D s}=\xi \frac{D X^{i}}{D s}+X^{i} \frac{d \xi}{d s} \text {. }
$$

It follows from the remarks made above that the vector $D X^{i} / D s$ is also normal with respect to the tangent vector $x^{i}$ of $E_{1}$ at $P$.

The equation of geodesic deviation was found to assume the following form ([1], p. 8):

$$
\frac{D^{2} \xi^{i}}{D s^{2}}=-R_{k h j}^{i}\left(x, x^{\prime}\right) \xi^{h} x^{\prime k} x^{\prime j},
$$

where the $R_{k_{h j}}^{i}$ are defined by the equations

$$
R_{k h j}^{i}=\frac{\partial P_{j k}^{i}}{\partial x^{h}}-\frac{\partial P_{h k}^{i}}{\partial x^{j}}+P_{h l}^{i} P_{j k}^{l}-P_{l k}^{i} P_{h j}^{l}+\frac{\partial P_{h k}^{i}}{\partial x^{\prime l}}\left\{\begin{array}{c}
l \\
p j
\end{array}\right\} x^{\prime p}
$$

Here the $P_{h k}^{i}$ are the generalised «coefficients of affine connection» (Cf. [3], p. 123), defined by

$$
P_{h k}^{i}\left(x, x^{\prime}\right)=\left\{\begin{array}{c}
i \\
h k
\end{array}\right\}_{\left(x, x^{\prime}\right)}-\frac{1}{2} g^{i m}\left(x, x^{\prime}\right) \frac{\partial g_{h m}\left(x, x^{\prime}\right)}{\partial x^{\prime l}}\left\{\begin{array}{c}
l \\
p k
\end{array}\right\}_{\left(x, x^{\prime}\right)} x^{\prime p}
$$


It is now our aim to derive from equations (1.4) a single differential equation of the second order in $\xi$. In order to do so we note that $E_{1}$ and $E_{2}$ define a two-dimensional geodesic subspace $G_{2}$ of $F_{n}$, whose "pole» is located at $O$, the point of intersection of $E_{1}$ and $E_{2}$. We denote the coordinates of $G_{z}$ by $u^{\alpha}(\alpha, \beta=1,2)$, and the components of $X^{i}$ with respect to $G_{2}$ by $U^{\alpha}$, since, by definition, $X^{i}$ is a vector of $G_{2}$. The (induced) Finslen metric $\bar{g}_{\alpha \beta}$ of $G_{2}$ defines an induced» covariant derivative (Cf. [9], \& 3 ) in $G_{2}$, an operation to be denoted by $\delta$. The components of the unit tangent vector to $E_{1}$ are written as $u^{\prime \alpha}$.

Our theory rests on the following fundamental

Theorem: When $X^{i}$ is a nnit vector defined according to (1.2), we have

$$
\frac{\delta U^{x}}{\delta s}=0
$$

as we differentiate covariantly with respect to the metric of $G_{2}$ along the geodesic $E_{\mathrm{a}}$ (although in general $D X^{i} / D s \neq 0$ ); i. e. the unit variation vector is transported by parallel displacement relative to $G_{z}$ along $E_{1}$.

Proof. - Let us consider first the case $n=3$. The geometrical relations are most easily grasped for this case, and the corresponding generalisation for an arbitrary $n$ can be written down immediately. We denote by $n^{* i}\left(x, x^{\prime}\right)$ the unit generator of the "normal cone» of $G_{2}$ at $P$ corresponding to the direction $x^{\prime i}$ (Cf. [9], $\left.\$ 4\right)$; i. e. $n^{* i}\left(x, x^{\prime}\right)$ satisfies the equations

$$
\begin{gathered}
g_{i j}\left(x, x^{\prime}\right) \frac{\partial x^{i}}{\partial u^{\alpha}} n^{* j}\left(x, x^{\prime}\right)=0, \\
g_{i j}\left(x, n^{*}\left(x, x^{\prime}\right)\right) n^{* i}\left(x, x^{\prime}\right) n^{* j}\left(x, x^{\prime}\right)=1 .
\end{gathered}
$$

Then the fundamental relation between $D X^{i} / D s$ and $\delta U^{\alpha} / \delta s$ can be expressed as follows (Cf. [10], p. 208):

$$
\frac{D X^{i}}{D s}=\left[\Omega_{\alpha \beta}^{*}(u r) U^{\alpha} u^{\prime \beta}\right] n^{* i}\left(x, x^{\prime}\right)+\frac{\partial x^{i}}{\partial u^{\alpha}} \cdot \frac{\delta U^{\alpha}}{\delta s},
$$

where the $\Omega_{\alpha \beta}^{*}(u r)$ are the coefficients of the «secondary" second fundamental form of $G_{2}$ at $P$ (Cf. [10], \& 7). Now, since $x^{i}=\left(\frac{\partial x^{i}}{\partial u^{\alpha}}\right) u^{\prime \alpha,}$, we find by multiplying (1.8, by $u^{\prime \alpha}$ and summing over $\alpha$, that

$$
g_{i j}\left(x, x^{\prime}\right) x^{\prime} n^{* i}\left(x, x^{\prime}\right)=0 .
$$

Hence it follows from $(1.10)$ that

$$
g_{i j}\left(x, x^{\prime}\right) x^{\prime j} \frac{D X^{i}}{D s}=g_{i j}\left(x, x^{\prime}\right) x^{\prime j} \frac{\partial x^{i}}{\partial u^{\alpha}} \cdot \frac{\delta U^{\alpha}}{\partial s} .
$$


But we have seen (equation 1.3)) that the left-hand side of this equation is zero; hence the vector $\left(\frac{\partial x^{i}}{\partial u^{\alpha}}\right)\left(\frac{\delta U^{\alpha}}{\delta s}\right)$ of $G_{2}$ is also normal with respect to the tangent vector $x^{i}$ of $E_{1}$ at $P$. Since normality in $F_{n}$ also implies normality with respect to the metric of the subspace (Cf. [9], p. 368), we deduce that

$$
\bar{g}_{a \beta}\left(u, u^{\prime}\right) u^{\beta} \frac{\delta U^{\beta}}{\delta s}=0 \text {. }
$$

For the same reason $U^{\alpha}$ is normal with respect to $u^{\prime \alpha}$ since $X^{i}$ is normal with respect to $x^{\prime i}$. Hence, since both $U^{\alpha}$ and $\delta U^{\alpha} / \delta s$ are both contained in $G_{2}$, it follows that

$$
\frac{\delta U^{x}}{\delta s}=\lambda U^{\alpha}
$$

where $\lambda$ is a scalar factor. Also, in view of (1.2),

$$
g_{\alpha \beta}\left(u, u^{\prime}\right) U^{\alpha} U^{\beta} \equiv U_{\alpha} U^{\alpha}=1,
$$

we find by covariant differentiation with respect to $s$ :

$$
\frac{\delta U_{\alpha}}{\delta s} U^{\alpha}=-U_{\alpha} \frac{\delta U^{\alpha}}{\delta s}
$$

But we have in virtue of $(1.15)$, since the $\bar{g}_{\alpha \beta}$ are homogeneous of degree zero in their directional arguments,

$$
\frac{\delta U_{\alpha}}{\delta s} U^{\alpha} \equiv \bar{g}_{\alpha \beta}\left(u, u^{\prime}\right) \frac{\delta U^{\beta}}{\delta s} U_{\alpha} \equiv U_{\alpha} \frac{\delta U^{\alpha}}{\delta s}
$$

noting that $\delta g_{\alpha \beta}\left(u, u^{\prime}\right)=0$ along the geodesic $E_{1}$.

From (1.16), (1.14) and (1.15) we thus deduce

$$
0=U_{\alpha} \frac{\delta U^{\alpha}}{\delta s}=\lambda U_{\alpha} U^{\alpha}=\lambda \text {. }
$$

Glancing at (1.14) we see that this proves the theorem (1.7).

Furthermore, it then follows from (1.10) that the vector $D X^{i}, D s$ is normal with respect to $G_{2}$. If we denote the length of this vector by $f$, we deduce from (1.10) also that

$$
f=\left|\frac{D X}{D s}\right|=\Omega_{\alpha \beta}^{*}\left(u^{\varphi}\right) U \alpha u^{\prime \beta}, \quad \text { or } \quad \frac{D X^{i}}{D s}=f n^{* i}\left(x, x^{\prime}\right),
$$

since $n^{* i}$ is defined to be a unit vector.

We introduce two further scalar functions $\bar{X}$ and $\bar{n}$ by the equations

$$
\begin{gathered}
g_{i j}\left(x, x^{\prime}\right) n^{* i}\left(x, x^{\prime}\right) n^{* j}\left(x, x^{\prime}\right)=\bar{n}^{2}\left(x, x^{\prime}\right), \\
g_{i j}(x, X) X^{i} X^{j}=\bar{X}^{2} .
\end{gathered}
$$


Geometrically these quatities represent simply the ratio of the length ont off from the vector $X^{i}$ (or $n^{* i}$ ) by the ellipsoid $g_{i j}\left(x, x^{\prime}\right) p^{i} p^{j}=1$ ( $x^{i}$ fixed, $p^{i}$ variable) in $T_{n}(P)$ to the length cut off from $X^{i}$ (or $n^{* i}$ ) by the indicatrix $g_{i j}\left(x, x^{\prime}, x^{\prime i} x^{\prime j}=1\right.$ in $T_{n}(P)$. Using (1.17) and (1.18) we see that

$$
g_{i j}\left(x, x^{\prime}\right) \cdot \frac{D X^{i}}{D s} \cdot \frac{D X^{j}}{D s}=f^{2} \overline{n^{2}}\left(x, x^{\prime}\right)
$$

Also, since $X^{i}$ is a vector of $G_{2}$, it can be represented in the form

$$
X^{i}=\sum_{\alpha=1}^{2} \mu_{(x)} \frac{\partial x^{i}}{\partial x^{\alpha}}
$$

the $\mu_{(x)}$ being scalar factors, while the $\partial x^{i} / \partial u^{\alpha}$ are the components of the tangent vectors to the parameter curves $u^{\alpha}=$ const. of $G_{2}$. Multiplying (1.8) by $\mu_{(x)}$ and summing over $\alpha$ (noting that not both the $\mu_{(\alpha)}$ ean vanish simultaneously), we find firstly that

$$
g_{i j}\left(x, x^{\prime}\right) X^{i} n^{* j}\left(x, x^{\prime}\right)=0,
$$

and secondly, in virtue of (1.17), since $f \neq 0$, that

$$
g_{i j}\left(x, x^{\prime}\right) X^{i} \frac{D X^{j}}{D s}=0
$$

Let us differentiate this equation covariantly with respect to $s$ along $E_{1}$, noting that $D g_{i j}\left(x, x^{\prime}\right) / D s=0$ since $x^{i}$ is the tangent vector to $E_{1}$ (Cf. [3], p. 122). We thus obtain

$$
\left.g_{i j}{ }^{\prime} x, x^{\prime}\right) \frac{D X^{i}}{D s} \cdot \frac{D X^{j}}{D s}=-g_{i j}\left(x, x^{\prime}\right) X^{i} \frac{D^{2} X^{j}}{D s^{2}}=f^{2} \widehat{n^{2}}\left(x, x^{\prime}\right)
$$

in virtue of (1.20:.

Now we differentiate equation (1.3) covariantly with respect to $s$ along $E_{1}$,

$$
\frac{D^{2} \xi^{i}}{D s^{2}}=\xi \frac{D^{2} X^{i}}{D s^{2}}+2 \frac{d \xi}{d s} \cdot \frac{D X^{i}}{D s}+X^{i} \frac{d^{2} \xi}{d s^{2}},
$$

and we multiply each side of this equation by $g_{i j}\left(x, x^{\prime}\right) X^{j}$ and sum over $i$, thus obtaining

$$
g_{i j}\left(x, x^{\prime}\right) X^{j} \frac{D^{2} \xi^{i}}{D s^{2}}=-f^{2} \widehat{n^{2}}\left(x, x^{\prime}\right) \xi+\frac{d^{2} \xi}{d s^{2}}
$$

when we observe (1.23), (1.22) and (1.19). Since the $x^{\prime i}, X^{i}$ are unit vectors, we call the quantity

$$
\widehat{K}\left(x, x^{\prime}, X\right) \equiv g_{i j}\left(x, x^{\prime}\right) X^{j} R_{k h r}^{i}\left(x, x^{\prime}\right) X^{h} x^{\prime k} x^{\prime r}
$$

the "scalar curvature» of $F_{n}$ for the directions $X^{i}, x^{i}$ with respect to $x^{i}\left({ }^{2}\right)$. Then, multiplying (1.4) by $g_{i j}\left(x, x^{j}\right) X^{j}$ and again summing over $i$, we find,

(i) Note that this is not equivalent to the $K$ defined in [1], $\$ 3$. 
taking note of (1.2)

$$
g_{i j}\left(x, x^{\prime}\right) X^{j} \frac{D^{2} \xi_{i}}{D s^{2}}=-\xi \widehat{K}\left(x, x^{\prime}, X\right)
$$

Comparing this equation to (1.25) and noting (1.17) we finally obtain the desired relations

$$
\frac{d^{2} \xi}{d s^{2}}+\xi\left[\widehat{K}\left(x, x^{\prime}, X\right)-\left[\Omega_{\alpha \beta}^{*}(u ̛) u^{\prime \alpha} U^{\beta}\right]^{2} \bar{n}^{2}\left(x, x^{\prime}\right)\right]=0 .
$$

These are the differential equations of second order for the length $\xi$ of the infinitesimal variation vector for the case $n=3$. It follows from the above argument that they are still valid for any value of $n$ when written in the form

$$
\frac{d^{2} \xi}{d s^{2}}+\xi\left[\widehat{K}\left(x, x^{\prime}, X\right)-f^{2} \bar{n}^{2}\left(x, x^{\prime}\right)\right]=0
$$

except that now we have to replace $f$ and $\bar{n}$ as follows:

We choose a set of $(n-2)$ independent solutions $n_{(y)}^{* i}\left(x, x^{\prime}\right)$ of $(1.8)$, whose existence can be proved without difficulty (Cf. [9], p. 372), in such a manner that the equations

$$
g_{i j}\left(x, \frac{D X}{D s}\right) n_{(\nu)}^{* i}\left(x, x^{\prime}\right) n_{(\mu)}^{* j}\left(x, x^{\prime}\right)=\delta_{\nu}^{\mu} \quad(\mu, \nu=3, \ldots, n)
$$

are satisfied. To each $n_{(y)}^{* i}\left(x, x^{\prime}\right)$ a tensor $\Omega_{(y) \times \beta}^{*}$ of rank two can be associated (in a manner exactly analogous to that expounded in $\$ 7$ of [10]), and equations (1.10) now become

$$
\frac{D X^{i}}{D s}=\sum_{\nu=3}^{n}\left[\Omega_{(\nu) \alpha \beta}^{*}(u \Upsilon) u^{\prime \alpha} U^{\beta}\right] n_{(\nu)}^{* i}\left(x, x^{\prime}\right)+\frac{\partial x^{i}}{\partial u^{x}} \cdot \frac{\delta U^{\alpha}}{\delta s} .
$$

The rest of the argument, showing $\delta U^{\alpha} / \delta s=0$, runs as before with $n_{(v)}^{* i}$ replacing $n^{* i}$ in (1.11). From $\left(1.10^{\prime}\right)$ we then deduce

$$
f^{2} \equiv g_{i j}\left(x, \frac{D X}{D s}\right) \frac{D X^{i}}{D s} \cdot \frac{D X^{j}}{D s}=\sum_{\mu=3}^{n} \sum_{y=8}^{n}\left(\mathbf{Q}_{(\mu) \times \beta}^{*} u^{\prime x} U^{\beta}\right)\left(\Omega_{(j) x \beta}^{*} u^{\prime \alpha} U^{\beta}\right) g_{i j}\left(x, \frac{D X}{D s}\right) n_{(p)}^{* i} n_{(j)}^{* j},
$$

or, in virtue of condition $\left(1.9^{\prime}\right)$

$$
f=+\left[\sum_{\mu=3}^{n}\left(Q_{(v) \alpha \beta}^{*} u^{\prime \alpha} U^{\beta}\right)^{q^{2}}\right]^{1 / 2},
$$

and finally we have to put

$$
\bar{n}^{*}\left(x, x^{\prime}\right)=\frac{g_{i j}\left(x, x^{\prime}\right) \sum_{y=3}^{n} \sum_{\nu=8}^{n}\left(\Omega_{(y) x \beta}^{*} u^{\prime} \times U^{\beta}\right)\left(\Omega_{(\mu) \times \beta}^{*} u^{\prime \alpha} U^{\beta}\right) n_{(\nu)}^{* i}\left(x, x^{\prime}\right) n_{(u)}^{* j}\left(x, x^{\prime}\right)}{\sum_{y=3}^{n}\left(\Omega_{(v) \times \beta}^{*} u^{\prime \alpha} U^{(\beta}\right)^{2}} .
$$


Substituting in this equation from $\left(1.10^{\prime}\right)$ and $\left(1.17^{\prime}\right)$ we see that $(1.20)$ remains satisfied. Equation (1.21) is now replaced by the set of $(n-2)$ equations:

$$
g_{i j}\left(x, x^{i}\right) X^{i} n_{(v)}^{* j}\left(x, x^{\prime}\right)=0 .
$$

Multiplying this by $\Omega_{(y) \times \beta}^{*}\left(u_{Y}\right) u^{\prime} x^{\prime \beta}$ and summing over $\gamma$, we obtain once more equation (1.22)- on observing (1.10'). The rest of the argument follows as before.

In order to compare these results with the known facts of Riemannian Geometry (i. e. $g_{i j}\left(x, x^{\prime}\right)$ independent of $x^{\prime}$ ) we remark that $\bar{n} \equiv 1, \bar{X}=X \equiv 1$. Denoting the «associated curvature» (curvatura associate) in the direction of $X^{i}$ by $\left(R_{X}\right)^{-1}$, we find that

$$
\frac{1}{R_{X}^{2}}=\sum_{\nu=2}^{n}\left(\Theta_{(\nu) \times \beta} u^{\prime \alpha} U^{\beta}\right)^{2}
$$

where the $\Omega_{(0) \times \beta}$ represent the coefficients of the usual second fundamental form of the two-dimensional subspace of the Riemannian space ander consideration; and equations (1.28) reduce to the form

$$
\frac{d^{2} \xi}{d s^{2}}+\xi\left[K_{x^{\prime}, X}-\frac{1}{R_{X}^{2}}\right]=0
$$

where $K_{x^{\prime}, X}$ is the Riemannian curvature of the space for the 2-plane defined by $x^{\prime}$ and $X^{i}$. This form of the equations of geodesic deviation was given by ONICESCU ([5], p. 562) and Bortolotti ([6], p. 13).

In the case of two dimensions it follows from (1.7) that $D X / D s=0$, so that $f=0$ in virtue of (1.20), and equation (1.28) reduces to (Cf. [1], eqn. (4.10))

$$
\frac{d^{2} \xi}{d s^{2}}+\xi \bar{K}\left(x, x^{\prime}, X\right)=0
$$

This a generalisation to two-dimensional FINSLER spaces of JACOBI's equations in the theory of surfaces. It can be proved (Cf. [11], p. 216) for the case of a two-dimensional Riemannian space that these equations can also be obtained by writing down the EULER equations representing the necessary conditions that the integral of the second variation of the length integral of this $V_{2}$ taken along $E_{1}$ shall assume an extreme valne. This justifies the nomenclature "JACOBI' s Equations". In order to discuss the validity of this result for entirely general problems in the Calculus of Variations, irrespective of the number of dimensions, we shall have to evaluate the second variation of the integral of $F\left(x, x^{\prime}\right)$ taken along $E_{1}, F\left(x, x^{\prime}\right)$ being the metric function of our $F_{n}$. This naturally leads to a discussion of the accessory problem in the Calculus of Variations. 


\section{§ 2. - The second Variation of the length-Integral.}

In order to deal efficiently with an explicit evaluation of second variation, it is necessary to differentiate covariantly with respect to a parameter which is not the arc-length of the curve under consideration. For this purpose it was found neccessary to introduce new functions $P_{h k}^{* i}\left(x, x^{\prime}\right)$ which are generalisations of the $P_{h k}^{i}\left(x, x^{\prime}\right)$. We define the generalised covariant derivative $\left({ }^{3}\right)$ of a vector-field $X^{i}\left(x^{h}\right)$ with respect to an arbitrary parameter $t$ as follows:

$$
\frac{D X^{i}}{D t}=\frac{\partial X^{i}}{\partial t}+P_{h k}^{* i}\left(x, x^{\prime}\right) X^{h} \frac{\partial x^{k}}{\partial t},
$$

such that when $t=s$ (i. e. arc-length of curve) this reduces to the covariant derivative used above; this requires that we must have

$$
P_{h k}^{* i}\left(x, x^{\prime}\right) x^{\prime k}=P_{h k}^{i}\left(x, x^{\prime}\right) x^{\prime k}, \quad\left(x^{\prime k} \equiv d x^{k} / d s\right) .
$$

The analytic form of the $P_{h k}^{* i}$ is given by

$$
P_{h k, i}^{*}\left(x, x^{\prime}\right)=[h k, i]_{\left(x, x^{\prime}\right)}+[h k, i]_{\left(x, x^{\prime}\right)}^{(2)}+[h k, i]_{\left(x, x^{\prime}\right)}^{(8)},
$$

where the first bracket represents the usual CHRistoffer symbols of the first kind and the second and third brackets are formed in precisely the same manner by oyclic interchange of the indices of the quantities defined respectively by

(2.4) $\stackrel{(1)}{\Gamma}_{j k, h} \equiv-\frac{\partial g_{j k}\left(x, x^{\prime}\right)}{\partial x^{\prime l}}\left\{\begin{array}{c}l \\ p h\end{array}\right\} \quad x_{\left(x, x^{\prime}\right)}^{x^{p}} ; \quad \stackrel{(2)}{\Gamma}_{j k, h} \equiv-\frac{1}{2} \frac{\partial g_{j k}\left(x, x^{\prime}\right)}{\partial x^{\prime i}} g^{i m}\left(x, x^{\prime}\right) \stackrel{1}{\Gamma}_{h m, n} x^{\prime n}$.

These quantities may be derived in a simple geometrical manner and ensure that (2.1) is a vector. The homogeneity properties of the metric tensors leads to the statement (2.2). In virtue of this equation we may use the $P_{h k}^{* i}$ instead of the $P_{h k}^{i}$, even when we are differentiating with respect to the arc-length. We note that the $P_{h k}^{* i}$ are symmetrical in the lower indices.

In evaluating the first and second variations of the length-integral we shall follow a construction due to SxNGE for the case of a Riemannian space [7]. Let $C$ be a curve of $F_{n}$ joining two points $P_{1}$ and $P_{2}$. The family of admissible curves is denoted by

$$
x^{i}=x^{i}(u, v) \text { with } \frac{d u}{d s}=1 \text { along } C,
$$

where $s$ is the arc-length measured along each curve $v=$ const. from the intersection of these curves with the curve $u=u_{1}, P_{1}$ and $P_{2}$ being the points $\left(u_{1}, 0\right),\left(u_{z}, 0\right)$ respectively. $C$ is the curve $v=0$.

(3) A detailed geometrical construction, leading to the functions $P_{h k}^{*}, i$ and containing the proofs of statements made below in the present paper will be published shortly in a memoir entitled Ueber die Krümmungstensoren allgemeiner metriseher Räume. 
Let $F\left(x, x^{\prime}\right)$ be the distance function of $F_{n}$, so that

$$
F^{2}\left(x, x^{\prime}\right)=g_{i j}\left(x, x^{\prime}\right) x^{i} x^{\prime j} ; \quad g_{i j}\left(x, x^{\prime}\right)=\frac{1}{2} \frac{\partial^{2} F^{2}\left(x, x^{\prime}\right)}{\partial x^{\prime i} \partial x^{\prime j}} .
$$

For the arc-length of $C$ joining $P_{1}$ and $P_{2}$ we then have on using (2.5)

$$
L(v)=\int_{u_{1}}^{u_{2}}\left[F\left(x, x^{\prime}\right)\right]_{v=0} d u
$$

and the first variation of $C$ is written in the form

$$
\delta L=\delta v \int_{u_{1}}^{u_{3}}\left[\frac{\partial F}{\partial v}\right]_{v=0} d u .
$$

Noting (9.5) let us put

$$
\frac{\partial x^{i}(u, 0)}{\partial u}=x^{\prime i}, \quad \frac{\partial x^{i}(u, 0)}{\partial v}=\eta^{i} ;
$$

$\eta^{i}$ is called the variation vector, the infinitesimal variation vector being given by

$$
\xi^{i}=\eta^{i} \delta v
$$

From (2.9) we deduce

$$
\frac{\partial x^{\prime i}}{\partial v}=\frac{\partial \eta^{i}}{\partial u}
$$

Condition (2.5) implies that $F\left(x, x^{\prime}\right)=1$ along $C$. Thus we have, using (2.11) and (2.6)

$$
F \frac{\partial F}{\partial v}=\frac{\partial F}{\partial x^{k}} \eta^{k}+\frac{\partial F}{\partial x^{\prime i}} \cdot \frac{\partial \eta^{i}}{\partial u}=\frac{1}{2} \frac{\partial g_{j k}\left(x, x^{\prime}\right)}{\partial x^{h}} x^{\prime j} x^{\prime k} \eta^{h}+g_{i j}\left(x, x^{\prime}\right) \frac{\partial \eta^{i}}{\partial u} x^{\prime j},
$$

where we have made use of the homogeneity relations

$$
\frac{\partial g_{i j}\left(x, x^{\prime}\right)}{\partial x^{\prime k}} x^{\prime i}=\frac{\partial g_{i k}\left(x, x^{\prime}\right)}{\partial x^{\prime j}} x^{\prime j}=0 .
$$

Noting the well-known relations

$$
\frac{\partial g_{j k}}{\partial x^{h}} \equiv[j h, k]+[k h, j], \quad g_{i j}\left\{\begin{array}{c}
i \\
k h
\end{array}\right\} \equiv[k h, j],
$$

we find

$$
F \frac{\partial F}{\partial v}=g_{i j}\left(x, x^{\prime}\right) x^{\prime j}\left[\frac{\partial \eta^{i}}{\partial u}+\left\{\begin{array}{c}
i \\
h k
\end{array}\right\}_{\left(x, x^{\prime}\right)} \eta^{h} x^{\prime k}\right],
$$


or on applying (2.12) to (1.6) and remembering (2.2)

$$
F \frac{\partial F}{\partial v}=g_{i j}\left(x, x^{\prime}\right) x^{\prime j}\left[\begin{array}{l}
\partial \eta^{i} \\
\partial u
\end{array}+P_{h k}^{* i}\left(x, x^{\prime}\right) \eta^{h} x^{\prime h}\right]=g_{i j}\left(x, x^{\prime}\right) x^{j} \frac{D \eta^{i}}{D u} .
$$

In virtue of $(2.6)$ we thus bave for the first variation:

$$
\delta L=\delta v \int_{u_{1}}^{u_{2}} g_{i j}\left(x, x^{\prime}\right) x^{\prime} j \frac{D \eta^{i}}{D u} d u=\delta v \int_{u_{1}}^{u_{2}}\left|\frac{D \eta}{D u}\right| \cos \left(x^{\prime}, \frac{D \eta}{D u}\right) d u,
$$

the (unsymmetrical) Minkowskian cosine being defined as in [8], § 2. This form of the first variation is a generalisation of the one obtained by SxNGE (loc. eit.) for Riemannian spaces, using, however, a different mode of calculation. The following theorems result in the same manner:

(1) The first variation vanishes if the covariant derivative of the variation vector is normal (i. e. transversal) with respect to the tangent-vector of $C$.

(2) The first variation vanishes if the variation vector is transported by parallel displacement along $C$.

(3) The first variation is positive or negative according as the angle $\Theta\left(x^{\prime}, D \eta / D u\right)$ is acute or obtuse.

NoтE. - The angle $\Theta\left(\lambda^{i}, \mu^{i}\right)$ between the unit vectors $\lambda^{i}, \mu^{i}$ is said to be acute (obtuse) if $\mu^{i}$ has to be produced in its positive (negative) direction in order to meet the tangent-hyperplane of the surface $F\left(x, x^{\prime}\right)=1$ drawn at the point $\lambda^{i}$ of this surface. If it does not meet this plane when produced in either directions the vector $\mu^{i}$ is said to be normal with respect to the vector $\lambda^{i}$.

Now, if we differentiate along $C$, we have $D g_{i j}\left(x, x^{\prime}\right) / D s=0$ so that

$$
g_{i j}\left(x, x^{\prime}\right) x^{\prime j} \frac{D \eta^{i}}{D u}=\frac{D}{L u}\left(g_{i j}\left(x, x^{\prime}\right) x^{\prime i} \eta^{j}\right)-g_{i j}\left(x, x^{\prime}\right) \eta^{j} \frac{D x^{\prime i}}{D u} \text {. }
$$

and (2.5) assumes the alternative form:

$$
\delta L=\delta v\left\{\left[g_{i j}\left(x, x^{\prime}\right) x^{i \eta_{\eta}} \eta^{j}\right]_{u_{1}}^{u_{2}}-\int_{u_{1}}^{u_{2}} g_{i j}\left(x, x^{\prime}\right) \eta^{j} \frac{D x^{i}}{D u} d u\right\} .
$$

A geodesic is characterised by the condition of autoparallelism: $D x^{\prime i} / D s=0$ (vanishing of principal normal), so that we have the additional result:

(4) The first variation vanishes if $C$ is a geodesic and if either the variation vector vanishes at the end-points or is normal with respect to the tangent vector of $C$ at the end-points.

From (2.8) we have for the second variation:

$$
\delta^{2} L=\frac{1}{2} \delta v^{2} \int_{u_{3}}^{u_{2}}\left[\frac{\partial^{2} F}{\partial v^{2}}\right]_{v=0} d u
$$


Differentiating equation (2.14) covariantly with respect to $v$ along $C$, we find

$$
\left(\frac{\partial F}{\partial v}\right)^{2}+F \frac{\partial^{2} F}{\partial u^{2}}=g_{i j}\left(x, x^{\prime}\right) \frac{D x^{\prime j}}{D v} \cdot \frac{D \eta^{i}}{D u}+g_{i j}\left(x, x^{\prime}\right) x^{\prime j} \frac{D^{2} \eta^{i}}{D v D u}
$$

since $\left[D g_{i j}\left(x, x^{\prime}\right) / D v\right] x^{\prime}=0$ along $C$ (Cf. Footnote $\left.\left({ }^{8}\right)\right)$. Now we have in virtue of $(2.11)$

$$
\frac{D x^{\prime i}}{D v}=\frac{\partial x^{i}}{\partial v}+P_{h k}^{* i}\left(x, x^{\prime}\right) x^{\prime h} \frac{\partial x^{h}}{\partial v}=\frac{\partial \eta^{i}}{\partial u}+P_{h k}^{* i}\left(x, x^{\prime}\right) \eta^{k} \frac{\partial x^{h}}{\partial u},
$$

since the $P_{h i}^{* i}$ are symmetrical in $h, k$. H nce it follows that we have along $C$

$$
\frac{D x^{i}}{D v}=\frac{D \eta^{i}}{D u}
$$

a result that is corroborated by a glance at (2.2). Substituting this and (2.14) in (2.19), noting that $F=1$, we find for (2.18)

$$
\begin{gathered}
\delta^{2} L=\frac{1}{2} \delta v^{2} \int_{u_{1}}^{u_{2}}\left(\left[g_{i j}\left(x, x^{\prime}\right)-g_{i k}\left(x, x^{\prime}\right) g_{j h}\left(x, x^{\prime}\right) x^{\prime h} x^{\prime h}\right] \frac{D \eta^{i}}{D u} \cdot \frac{D \eta^{g}}{D u}\right. \\
\left.+g_{i j}\left(x, x^{\prime}\right) x^{\prime j} \frac{D^{2} \eta^{i}}{D v D u}\right) d u
\end{gathered}
$$

This is the first form of the second variation, and it represents once more a generalisation of a similar result due to SYNGE (loc. cit.) for the case of a Riemannian space. Again the mode of deduction is an entirely different one. We note that we can also write $(2.21)$ in the form:

$$
\delta^{2} L=\frac{1}{2} \delta v^{2} \int_{u_{1}}^{u_{2}}\left(g_{i j}\left(x, x^{\prime}\right) \frac{D \eta^{i}}{D u} \cdot \frac{D \eta^{j}}{D u}-\cos ^{2}\left(x^{\prime}, \frac{D \eta}{D u}\right)\left|\frac{D \eta}{D u}\right|^{2}+g_{i j}\left(x, x^{\prime}\right) x^{\prime j} \frac{D^{2} \eta^{i}}{D v D u}\right) d u
$$

remarking at the same time that in Minkowskian trigonometry (Cf. [8], \& 2) the relation $\cos ^{2}\left(x, y^{\prime}\right)=1-\sin ^{2}\left(x^{\prime} y^{\prime}\right)$ is not in general true. Hence we are not in a position to generalise SYNGE' $s$ theorems as regards the sign of the second variation. This has to be determined by direct inspection of (2.22) in each particular case. We merely note that

(5) The second variation of the length-integral vanishes if the variation vector is transported by parallel diplacement along $C$.

We shall now discuss the second form of the second variation. In order to do so we note the following formula (Cf. Footnote $\left(^{3}\right)$ ): If $X^{i}\left(x^{k}\right)$ is an arbitrary continuous and continuously differentiable vector field of $F_{n}$ defined along the curve $C$, at each point of which two arbitrary directions $\xi^{r}=\partial x^{r} / \partial u$, $\eta^{r}=\partial x^{r} / \partial v$ are given by some geometrical consideration, we have quite generally

$$
\frac{D^{2} X^{i}}{D v D u}-\frac{D^{2} X^{i}}{D u D v}=K_{h r k}^{i}\left(x, x^{\prime}\right) X^{k} \eta^{r} \xi^{k}
$$


where we define the curvature tensor by the relations ( $\left.{ }^{4}\right)$

$$
K_{h r k}^{i}=\frac{\partial P_{h k}^{* i}}{\partial x^{r}}-\frac{\partial P_{h r}^{* i}}{\partial x^{k}}+P_{m r}^{* i} P_{h k}^{* m}-P_{m k}^{* i} P_{h r}^{* m}+\frac{\partial P_{h k}^{* i}}{\partial x^{\prime l}}\left(\frac{\partial x^{\prime l}}{\partial x^{r}}\right)_{u}-\frac{\partial P_{h r}^{* i}}{\partial x^{\prime l}}\left(\frac{\partial x^{\prime l}}{\partial x^{n}}\right)_{v}
$$

Here the terms $\left(\partial x^{\prime} / \partial x^{r}\right)_{u}$, $\left(\partial x^{\prime} / \partial x^{r}\right)_{v}$ are the derivatives of the tangent vector of (2.5) in the $v$ and $u$ directions respectively. These are determined by the particular problem under consideration - this is a geometrical peculiarity of Finsler spaces. Thus when two expressions arising from different problems involving the curvature tensor are being compared with each other, one has to establish the identity of meaning of these two functions before one can draw any comparisons.

In order to apply (2.23) to (2.21) we put $X^{i}=\eta^{i}$ and $\xi^{n}=x^{k}$ in the former formula, thus obtaining

$$
\begin{aligned}
\delta^{2} L & =\frac{1}{4} \frac{1}{2} \delta v^{2} \int_{u_{k}}^{u_{2}}\left\{\left(g_{i j}\left(x, x^{\prime}\right)-g_{i k}\left(x, x^{\prime}\right) g_{j h}\left(x, x^{\prime}\right) x^{\prime h} x^{\prime k}\right) \frac{D \eta^{i}}{D u} \cdot \frac{D \eta^{j}}{D u}\right. \\
& \left.+g_{i j}\left(x, x^{\prime}\right) x^{\prime} j K_{h r k}^{i}\left(x, x^{\prime}\right) \eta^{h} \eta^{r} x^{\prime k}+g_{i j}\left(x, x^{\prime}\right) x^{\prime j} \frac{D^{2} \eta^{i}}{D u D v}\right\} d u
\end{aligned}
$$

Now

$$
g_{i j}\left(x, x^{\prime}\right) x^{j j} \frac{D^{2} \eta^{i}}{D u D v}=\frac{D}{D u}\left(g_{i j}\left(x, x^{\prime}\right) x^{\prime j} \frac{D \eta^{i}}{D v}\right)-g_{i j}\left(x, x^{\prime}\right) \frac{D x^{j} j}{D u} \cdot \frac{D \eta^{i}}{D v}
$$

so that $(2.25)$ reduces to

$$
\begin{gathered}
\delta^{2} L=\frac{1}{2} \delta v^{2}\left[g_{i j}\left(x, x^{\prime}\right) x^{\prime j} \frac{D \eta^{i}}{D u}\right]_{u_{1}}^{u_{2}} \\
+-\frac{1}{2} \delta v^{2} \int_{u_{1}}^{u_{2}}\left\{\left(g_{i j}\left(x, x^{\prime}\right)-g_{i k}\left(x, x^{\prime}\right) g_{j h}\left(x, x^{\prime}\right) x^{\prime h} x^{\prime k}\right) \frac{D \eta^{i}}{D u} \cdot \frac{D \eta^{J}}{D u}\right. \\
-g_{i j}\left(x, x^{\prime}\right) \frac{D x^{\prime \prime}}{D u} \cdot \frac{D \eta^{i}}{D v}+K_{i h r k}\left(x, x^{\prime}\right) x^{\prime i} \eta^{h} x^{\prime k} \eta^{r} \mid d u,
\end{gathered}
$$

Equation (2.26) represents the second form of the second variation. When $C$ is a geodesic, (i. e. $D x^{\prime i} / D u=0$ ) this reduces to a form obtained by E. T. DA. VIES [12], using, however, the calculus of CARTAN and correspondingly different methods of deduction.

(4) A similar tensor has recently been obtained by E. Bompiani under more general conditions (Cf. [4], p. 184). 


\section{§ 3. - The Accessory Problem.}

In order to discuss the relationship of our second form of the second variation with the results obtained in $\S 1$, we shall now suppose that we are given a normal variation, i. e.

$$
g_{i j}\left(x, x^{\prime}\right) x^{\prime j} \eta^{i}=0 .
$$

Differentiating this equation covariantly with respect to $u$ along $C$, we have

$$
g_{i j}\left(x, x^{\prime}\right) \frac{D x^{\prime j}}{D u} \eta^{i}=-g_{i j}\left(x, x^{\prime}\right) x^{\prime j} \frac{D \eta^{i}}{D u} .
$$

Furthermore, we can identify $O$ with the geodesic $E_{1}$ of $\S 1$, thus putting $D x^{\prime j} / D u=0$; so that the expressions on either side of (3.2) vanish along $C$. It then follows that (2.26) reduces to the form

$$
\delta^{2} L=\frac{1}{2} \delta v^{2} \int_{u_{1}}^{u_{2}}\left\{g_{i j}\left(x, x^{\prime}\right) \frac{D \eta^{i}}{D u} \cdot \frac{D \eta^{j}}{D u}+K_{i h r k}\left(x, x^{\prime}\right) x^{\prime i} \eta^{h} x^{\prime k} \eta^{r}\right\} d u .
$$

Taking into account equations (2.10) and (1.2) we can write this in our original notation:

$$
\delta^{2} L=\frac{1}{2} \int_{u_{1}}^{u_{2}}\left\{g_{i j}\left(x, x^{\prime}\right) \frac{D \xi^{i}}{D u} \cdot \frac{D \xi^{j}}{D u}+\xi^{2} K_{i h r k}\left(x, x^{\prime}\right) x^{\prime i} X^{h^{\prime} x^{\prime k}} X^{\prime \prime}\right\} d u .
$$

The accessory problem can now be formulated as follows (Cf. [13], pp. 260-263): Regarding the (scalar) integrand of (3.4) as function of $u, \xi$ and $d \xi / d u$, those curves must be sought which minimise the integral (3.4), i. e. we have to establish the EuLer equations of the new problem in the Calculus of Variations represented by the integral (3.4). The principal result of this section can then be formulated in the following manner:

Theorem: The Euler equations of the accessory problem in terms of $\xi$ - the so-called JACOBI equations - contain the scalar form $\left(1.28^{\prime}\right)$ of the equations of geodesic deviation.

Before proving this theorem we note that it would not hold if we form the $(n-1)$ independent EULER equations of the accessory problem regarding the integrand $(3.4)$ as a function of $\xi^{i}$ and $d \xi^{i} / d u$, thus obtaining the JACOBI equations in tensor form. A brief caloulation shows that this is due to the fact that we cannot thus obtain the tensor equations (1.4) of geodesic deviation since the tensor $K_{i h r k}$ is not symmetrical in $h$ and $r$.

The first step in the proof is to show that the scalar quantities (1.26) and the last term of the integrand of (3.4) are equal (apart from the factor $\xi^{*}$ ) when the unit vectors $X^{i}$ have the same meaning in each case. To do so we note that the quantities $R_{k h j}^{i}$ defined in 1.5 ) could be replaced in (1.4) by 
the tensor (2.24). This step is easily verified in view of equations (2.12); for it is seen that the additional terins occuring in (2.24) would vanish identically when the product $K_{k h j}^{i} x^{\prime h} x^{\prime h}$ of (1.4) is formed. Alternatively, one could simply rewrite the calculation of [1], $\$ \S 2 \cdot 3$, using the $P_{h k}^{* i}$ instead of the $P_{h k}^{i}$, avoiding all simplifications that would result from (2.12) and (2.2). In this way one would obtain once more the tensor $K_{k h j}^{i}$ instead of $R_{k h j}^{i}$. Since these two processes are merely mechanical, we shall not give a detailed description here. Thus we still have to show that

$$
K_{i h r k}\left(x, x^{\prime}\right) x^{\prime i} X^{h} X^{r} x^{\prime k}=-K_{i h r k}\left(x, x^{\prime}\right) X^{i} x^{\prime h} X^{\prime \prime} x^{\prime k} .
$$

The skew-symmetry of the tensor $K_{i h r k}$ in $i$ and $h$ is not at all obvious in view of the last two terms on the right-hand side of (2.24); yet for our purposes it is sufficient to prove that

$$
K_{s h r k}\left(x, x^{\prime}\right) x^{\prime s} x^{\prime k}=-K_{h s r k} x^{\prime s} x^{\prime k},
$$

since from this equation the required result (3.5) will follow immediately on inner multiplication with $X^{h} X^{r}$ and interchange of dummy-suffixes $\left({ }^{5}\right)$. In order to prove (3.6) we choose special coordinates along $E_{1}$ as follows: Let $O$ be a fixed point of $E_{1}, P$ an arbitrary point of $E_{1}$ whose distance - defined as the arc-length $O P$ - from $O$ is $s$. If $x_{(0)}^{\prime i}$ denotes the tangent-vector to $E_{1}$ at $O, P$ is completely determined by the quantities $s$ and $x_{(0)}^{\prime i}$, so that we define the coordinates $z^{i}$ of $P$ by $z^{i}=x_{(0)}^{i}$ s. Since the differential equations of $E_{1}$ are then $d^{2} z^{i} / d s^{2}=0$, it follows that in these coordinates the quantities

$$
\left\{\begin{array}{c}
i \\
j k
\end{array}\right\}_{\left(x, x^{\prime}\right)} x^{\prime j} x^{\prime k}=0
$$

along $E_{1}$. Also, we note the analytical expression (Cf. Footnote $\left(^{3}\right)$ )

$$
\begin{aligned}
-K_{s h r k} & =\left(\frac{\partial P_{h k, s}^{*}}{\partial x^{r}}-\frac{\partial P_{h r, s}^{*}}{\partial x^{k}}\right)+\left(P_{s k}^{* j} P_{h r}^{* i}-P_{s r}^{* j} P_{h k}^{* j}\right) g_{i j} \\
& +\left(\frac{\partial P_{h k, s}^{*}}{\partial x^{\prime l}} \cdot\left(\frac{\partial x^{\prime}}{\partial x^{r}}\right)_{u}-\frac{\partial P_{h r, s}^{*}}{\partial x^{\prime l}} \cdot\left(\frac{\partial x^{\prime l}}{\partial x^{k}}\right)_{v}\right),
\end{aligned}
$$

(5) Footnote arlded during proof. This paper was already in print before the author succeded in establishing the following more general identity:

$$
K_{i h r k}\left(x, x^{\prime}\right) x^{\prime i}=-K_{k i r k i}\left(x, x^{\prime} \vdash x^{\prime},\right.
$$

of which (3.6) is a special case. The proof of this formula is derived by simply writing down the commutation formulae of the covariant derivatives of the $g_{i j}$. Furthermore, a straight-forward calculation shows that $\left(\partial P^{* i}{ }_{h k} / \partial x^{\prime}\right) x^{\prime \prime} x^{\prime k}=0$ in view of $(2.12)$, so that we deduce from (2.24) that $g_{i j} K^{i}{ }_{h r k} x^{\prime h} x^{\prime h} X^{j} X^{r}=\bar{K}$ is independent of the factor $\left(\partial x^{\prime l} / \partial x^{r}\right)_{u}$. Thus the rather laborious argument up to the paragraph ending above equation (3.11) is no longer necessarer. 
which hold along $E_{1}$. Using (2.3), (2.4), (2.12) and (3.7) we find in our new coordinates

$$
P_{h k, s}^{*} x^{\prime s}=[h k, s] x^{\prime s}, \quad P_{h r, s}^{*} x^{\prime r}=[h r, s] x^{\prime r} .
$$

Thus

$$
\begin{gathered}
\left(\frac{\partial P_{h k, s}^{*}}{\partial x^{r}}-\frac{\partial P_{h r, s}^{*}}{\partial x^{k}}\right) x^{s}=\left(\frac{\partial}{\partial x^{r}}[h k, s]-\frac{\partial}{\partial x^{k}}[h r, s]\right) x^{\prime s} \\
=-\left(\frac{\partial}{\partial x^{r}}[s k, h]-\frac{\partial}{\partial x^{k}}[s r, h]\right) x^{\prime s}
\end{gathered}
$$

the last step following in the same manner as in Riemannian Geometry. Hence we have

$$
\left(\frac{\partial P_{h k, s}^{*}}{\partial x^{r}}-\frac{\partial P_{h r, s}^{*}}{\partial x^{k}}\right) x^{\prime s} x^{\prime k}=-\left(\frac{\partial P_{s k, h}^{*}}{\partial x^{r}}-\frac{\partial P_{s r, h}^{*}}{\partial x^{k}}\right) x^{\prime s} x^{\prime k}
$$

in virtue of (3.9). Multiplying (3.9) by $x^{\prime k}$ and differentiating with respect to $x^{\prime l}$ we find in the same manner

$$
P_{h k, l}^{*} x^{\prime k}+P_{h l, s}^{*} x^{\prime s}+\frac{\partial P_{h k, s}^{*}}{\partial x^{\prime l}} x^{\prime s} x^{\prime k}=[h k, l] x^{\prime k}+[h l, s] x^{\prime s}+\frac{\partial}{\partial x^{\prime l}}[h k, s] x^{\prime s} x^{\prime k},
$$

noting that the first terms on either side cancel in view of (3.9). In view of the definition of the CHRISTOFFEL brackets and (2.12) it follows that

$\left(\frac{\partial P_{h k, s}^{*}}{\partial x^{\prime}} \cdot\left(\frac{\partial x^{\prime}}{\partial x^{r}}\right)_{u}-\frac{\partial P_{h r, s}^{*}}{\partial x^{\prime}}\left(\frac{\partial x^{\prime}}{\partial x^{k}}\right)_{v}\right) x^{\prime s} x^{\prime k}=-\frac{1}{2}\left(\frac{\partial^{z} g_{h k}}{\partial x^{\prime l} \partial x^{s}} \cdot\left(\frac{\partial x^{\prime}}{\partial x^{r}}\right)_{u}-\frac{\partial P_{h r, s}^{*}}{\partial x^{\prime}} \cdot\left(\frac{\partial x^{\prime}}{\partial x^{k}}\right)_{v}\right)^{\prime s} x^{\prime k}$.

In the latter expression the first term vanishes identically as a result of (2.12); remembering the meaning of the factor $\left(\partial x^{\prime} / \partial x^{k}\right)_{v}$ we see that the second term becomes

$$
\frac{1}{2} \frac{\partial P_{h r, s}^{*}}{\partial x^{\prime l}} \cdot \frac{d x^{\prime l}}{d s} \cdot x^{\prime s}=-\frac{1}{2} \cdot \frac{\partial P_{h r, s}^{*}}{\partial x^{\prime}}\left\{\begin{array}{c}
l \\
p j
\end{array}\right\} x^{\prime p} x^{\prime j} x^{\prime s}
$$

since $E_{\mathrm{t}}$ is a geodesic, and this term will also vanish in virtue of (3.7) in our particular coordinate system. In the same way we find

$\left(\frac{\partial P_{s k, h}^{*}}{\partial x^{\prime}} \cdot\left(\frac{\partial x^{\prime l}}{\partial x^{r}}\right)_{u}-\frac{\partial P_{s r, h}^{*}}{\partial x^{\prime l}} \cdot\left(\frac{\partial x^{\prime l}}{\partial x^{k}}\right)_{v}\right) x^{\prime s} x^{\prime k}=-\frac{1}{2}\left(\frac{\partial^{2} g_{s k}}{\partial x^{\prime} \partial r^{h}} \cdot\left(\frac{\partial x^{\prime}}{\partial x^{r}}\right)_{u}-\frac{\partial P_{s r, h}^{*}}{\partial x^{\prime}} \cdot\left(\frac{\partial x^{\prime l}}{\partial x^{k}}\right)_{v}\right) x^{\prime s} x^{\prime h}$,

and this expression will also vanish as a result of (2.12). Furthermore, we note that the skew-symmetry of the second expression in brackets on the right-hand side of (3.8) is obvions (as in Riemannian Geometry), so that due to the vanishing of the third expression (after inner multiplication with $x^{\prime} x^{\prime h}$ ) even after interchanging the indices $s$ and $h$, equation (3.6) resulls as a consequence of (3.10) in our special coordinate system, and, being a tensor equation, (3.6) must be true generally. 
Having proved (3.5), we can now write (3.4) in the following form, using (1.26):

$$
\delta^{2} L=\frac{1}{2} \int_{u_{1}}^{u_{2}}\left\{g_{i j}\left(x, x^{\prime}\right) \frac{D \xi^{i}}{D u} \cdot \frac{D \xi^{j}}{D u}-\xi^{2} \bar{K}\left(x, x^{\prime}, X\right)\right\} d u .
$$

If we denote once more by $X^{i}$ the unit vector in the direction of $\xi^{i}$, we see that the first term under the integral sign may be written in the form

$$
\xi^{2}\left(g_{i j}\left(x, x^{\prime}\right) \frac{D X^{i}}{D u} \cdot \frac{D X^{j}}{D u}\right)+2 \xi \frac{d \xi}{d u} g_{i j}\left(x, x^{\prime}\right) X^{i} \frac{D X^{j}}{D u}+\left(\frac{d \xi}{d u}\right)^{2} g_{i j}\left(x, x^{\prime}\right) X^{i} X^{j}
$$

Now equations (2.5) define a two-dimensional subspace $F_{2}$ of $F_{n}$ which contains the geodesic $E_{1}: v=0$ and the curve $v=\delta v=$ const. together with the vectors $\xi^{i}, X^{i}$. We can now apply the theory of $\S 1$ to this $F_{2}$ (noting the fact that nowhere in $\S 1$ use had been made of the fact that $G_{2}$ is a geodesic subspace), where, however, the symbols $n^{*}, \Omega_{\alpha \beta}^{*}$ now apply to $F_{2}$. This difficulty we avoid by once more denoting the length of the vector $D X^{i} / D s$ by $f$; and equation (1.22), is proved as before. Using (1.17) and (1.22) we see that the expression (3.12) becomes

$$
\xi^{2} f^{2} \bar{n}^{2}+\left(\frac{d \xi}{d s}\right)^{2}
$$

so that the second variation (3.11) reduces to the final normal scalar form

$$
\delta^{2} L=\frac{1}{2} \int_{s_{1}}^{s_{2}}\left\{\left(\frac{d \xi}{d s}\right)^{2}+\xi^{2}\left(f^{2} \widehat{n^{2}}-\widehat{K}\left(x, x^{\prime}, X\right)\right\} d s\right.
$$

in view of (2.5). Considering normal variations $\xi^{i}$ in an arbitrary fixed $F_{\mathrm{g}}$ defined in this manner, the integrand of (3.13) becomes a function of $s$, $\xi$ and $d \xi / d s$, since, by (1.17), $f^{*}$ and $K$ are completely determined by $F_{z}$. Treating this as a non-parametric problem in the Calculns of Variations, we see that the (scalar) JACOBI equations are

$$
\frac{d}{d s}\left\{2 \frac{d \xi}{d s}\right\}-\left\{f^{2} \widehat{n^{2}}-\widehat{K}\left(x, x^{\prime}, X\right)\right\} 2 \xi=0
$$

or, using (1.21),

$$
\frac{d^{\prime \prime} \xi}{d s^{2}}+\xi \mid \widehat{K}\left(x, x^{\prime}, X\right)-f^{2} \widehat{n}^{2} i=0 .
$$

But this equation is identical in form to the equation $\left(1.28^{\prime}\right)$ of geodesic deviation. We note that the quantities $f^{2}, \widehat{n^{2}}$ refer to $F_{2}$ and $G_{2}$ in (3.14) and $\left(1.28^{\prime}\right)$ respectively; hence for the special cas s that $F_{z}$ is a geodesic subspace 
the two equations have identical meaning. The theorem stated at the beginning of this section is therefore proved. For $n=2$ no distinction at all need be made between $F_{2}$ and $G_{2}$, so that in this case our result may be stated in the form that the scalar form of the equations of geodesic deviation is identical to the scalar $\mathrm{J}_{\mathrm{ACOBI}}$ equation. The variation curve $C^{*}$ satisfying (3.14) then satisfies the same differential equation as the geodesic $E_{2}$ of $\S 1$.

Suppose we carry out the above calculation of the second variation in such a manner as to restrict ourselves entirely to a given $F_{2}$ containing $C$, i. e. the normal variation vector must lie in $F_{2}$ and only intrinsic invariants of $F_{2}$ are used. Our results would be formally similar to (3.11), but we have to remember that the metric tensor, the covariant derivative and the scalar curvature now apply to $F_{2}$ :

$$
\delta^{2} L=\frac{1}{2} \int_{u_{1}}^{u_{2}}\left\{\bar{g}_{\alpha \beta}\left(u, u^{\prime}\right) \frac{\delta \xi^{\alpha}}{\delta u} \cdot \frac{\varepsilon \xi^{\beta}}{\delta u}-\xi^{2} \overline{\bar{K}}\left(u, u^{\prime}, U\right)\right\} d u,
$$

where $\hat{\bar{K}}\left(u, u^{\prime}, U\right)$ is the scalar curvature of $F_{2}$, and $U^{\alpha}$ is the unit vector in the direction of $\xi^{x}$. We have

$$
\frac{\delta \xi^{\alpha}}{\delta u}=\frac{d \xi}{d u} U^{\alpha}+\xi \frac{\delta U^{\alpha}}{\delta u}
$$

Since $\xi^{x}$ represents a normal variation, we have $\bar{g}_{\alpha \beta}\left(u, u^{\prime}\right) u^{\prime \beta} U^{\alpha}=0$ along $C$; and, since $C$ is a geodesic of $F_{2}$, we find by covariant differentiation

$$
\bar{g}_{\alpha \beta}\left(u, u^{\prime}\right) u^{\prime \beta} \frac{\delta U^{\alpha}}{\delta u}=0 \quad \text { or } \quad U^{\alpha}=\lambda \frac{\delta U^{\alpha}}{\delta u},
$$

where $\lambda$ is a scalar factor, since $U^{\alpha}$ and $\delta U^{\alpha} / \delta u$ are contained in the same two-dimensional subspace. We can now repeat the procedure followed in $\$ 1$ (equ. (1.14) et seq.), to prove $\delta U^{\alpha / \delta} u=0$. Using this result in (3.16) we find that $(3.15)$ reduces to

$$
\delta^{2} L=\frac{1}{2} \int_{u_{1}}^{u_{2}}\left\{\bar{g}_{\alpha \beta}\left(u, u^{\prime}\right) U^{\alpha} U^{\beta}\left(\frac{d \xi}{d u}\right)^{\mathbf{2}}-\xi^{2} \overline{\bar{K}}\left(u, u^{\prime}, \quad U\right)\right\} d u .
$$

But we also have (Cf. [9], § 1)

$$
\bar{g}_{\alpha \beta}\left(u, u^{\prime}\right) U^{\alpha} U^{\beta}=g_{i j}\left(x, x^{\prime}\right) \frac{\partial x^{i}}{\partial u^{\alpha}} \cdot \frac{\partial x^{j}}{\partial u^{\beta}} U^{\alpha} U^{\beta}=g_{i j}\left(x, x^{\prime}\left(X^{i} X^{j}=1\right.\right.
$$

by (1.2); substituting this result in (3.18) and noting that $\delta^{2} L$, being of the 1:ature of a length, must be the same whether calculated with respect to the metric of $F_{2}$ or the metric of the enveloping space $F_{n}$, we can regard this result as a special case of (3.13), thus obtaining

$$
\widehat{K}\left(x, x^{\prime}, X\right)-f^{2} \bar{n}^{2}=\widehat{\bar{K}}\left(u, u^{\prime}, U\right) \text {. }
$$


This equation gives us the relation between the scalar curvature of an $F_{2}$ along a geodesic $C$ contained in $F_{2}$ as compared with the scalar curvature of the enveloping space. The JACOBI equation (3.14) corresponding to variations restricted to our $F_{2}$ thus becomes

$$
\frac{d^{2} \xi}{d s^{2}}+\xi \overline{\bar{K}}\left(u, u^{\prime}, U\right)=0 .
$$

But in view of (3.5) this is precisely the scalar form (1.30) of the equations of geodesic deviation obtained for two-dimensional FINSLER spaces, and could therefore have been obtained by calculating *intrinsically " the deviation of two geodesics $C$ and $C^{*}$ of $F_{2}$ by the method of [1], $\$ \S 2-3$. Hence it follows from the theory of differential equations that solutions of the Jacobi equations (3.20) corresponding to variations restricted to a given two-dimensional subspace $F_{2}$ containing the geodesic $C$ are geodesics of that subspace in the neighbourhood of $C$.

It is of interest to note that we can carry out the integration (3.13) when we proceed along a solution of (3.14). For substituting from the latter equation in (3.13) we find

$$
\begin{aligned}
\delta^{2} L & =\frac{1}{2} \int_{s_{1}}^{s_{2}}\left[\left(\frac{d \xi}{d s}\right)^{2}+\xi\left(\frac{d^{2} \xi}{d s^{2}}\right)\right] d s \\
& =\frac{1}{2} \int_{s_{1}}^{s_{2}} \frac{d}{d s}\left(\xi \frac{d \xi}{d s}\right) d s
\end{aligned}
$$

so that we finally have

$$
\delta^{2} L=\frac{1}{2}\left[\xi \frac{d \xi}{d s}\right]_{s_{1}}^{s_{2}}
$$

which vanishes for fixed end-points (if such a solution of (3.14) exists).

In dealing with the question as to whether a geodesic $C$ will actually furnish us with a relative minimum of the length-integral, we must investigate the sign of $\delta^{2} L$. Assuming the WeImRstrass condition fulfilled (i. e. convexity of the iadicatrix) it follows from their definition that the quantities $\bar{X}^{2}, \bar{n}^{2}$, $f^{2}$ are positive, so that from (3.13) we can immediately deduce the following

THEOREM: The second variation along a geodesic $C$ is positive for all variations and $C$ will therefore furnish us with a relative minimum if the curvature $K\left(x, x^{\prime}, X\right)$ of $F_{n}$ is nowhere positive along $C$ for every twodimensional plane element $\left(x^{\prime}, X\right)$ containing the direction of $C$.

'This is a generalisation of a theorem of JACOBI for the case of twodimensional surfaces. (Cf. [15], p. 231). More specifically we would require

$$
\widehat{K}\left(x, x^{\prime}, X\right) \leq f^{2} \overline{n^{2}}
$$


at each point of $C$ for all unit normal vector fields $X^{i}$ of $C$, (noting that $X^{i}$ defines the corresponding $F_{2}$ and hence $\bar{n}^{2}$ ). This condition is less stringent than that required by the theorem but yields the same result. Our theorem is applicable to any problem in the Calculus of Variations in parametric form, provided the function $F\left(x, x^{\prime}\right)$ is differentiable up to and including the second and fourth order in $x^{k}$ and $x^{\prime k}$ respectively. For purposes of practical calcalation the expression for $\widehat{K}\left(x, x^{\prime}, X\right)$ can be simplified considerably by the use of equations (2.12) and the choice of special coordinate systems.

Comparing (3.22) with (3.19) we see that by constructing subspaces $F_{2}$ containing $C$ with negative scalar curvature $\overline{\bar{K}}\left(u, u^{\prime}, U\right)$ the second variation can be made positive for all normal variations constrained to lie in such an $F_{2}$.

Finally, using StuRM' s theorem ([11, p. 218), we see that if for any $\dot{F}_{2}$ containing $C$ there exists a positive constant $A$ such that $\bar{K}\left(u, u^{\prime}, U\right)>1 / A$ at all points of $C$, the distance between conjugate points (defined as usual by the zeros of solutions of (3.20) on $C$ for variations in $F_{2}$ will be less than $\pi \vee \bar{A}$. Now let $A^{*}=$ lower bound of all positive numbers $A$ obtained in this way by considering all possible subspaces $F_{2}$ containing $C$. Then $\pi \sqrt{A^{*}}$ is less than any other $\pi \sqrt{A}$, and using (3.19) and (1.20) we have the following THEOREM. - If there exists a positive constant $A^{*}$ such that

$$
\widehat{K}\left(x, x^{\prime}, X\right)-g_{i j}\left(x, x^{\prime}\right) \frac{D X^{i}}{D s} \cdot \frac{D X^{j}}{D s}>1 / A^{*},
$$

along a geodesic $C$ for all unit vector fields $X^{i}$ normal with respect to $C$, the distance betreen conjugate points on $C$ will be less than $\pi \sqrt{A^{*}}$.

In conclusion the following remarks may be of general interest. CARATHÉODORY succeeded in establishing the basic necessity conditions in the Calculus of Variations without the introduction of the first variation, using, instead, a direct method which is geometrically equivalent to the construction of orthogonal trajectories (i. e. transversals) to certain families of hypersurfaces in $F_{n}$. The results of the present paper show that further results (e.g. theory of conjugate points) can similarly be derived by purely geometrical considerations without the introduction of the second variation; for the type of problem treated above it is sufficient to study the deviation of geodesics of two-dimensional subspaces instead.

\section{BIBLIOGRAPHY}

[1] H. Ruvd, Eine Krümmungstheorie in Einslerschen Räumen, "Math. Ann ", 125, 1.18, (1952).

[2] T. Levi-Crvita, Sur l'écart géodésique, « Math. Ann.», 97, 291.320, (1926).

[3] H. Rund, Veber die Parallelverschiebung in Einsterschen Häumen, "Math. Z.", 54, 115̃-128, (1951). 
[4] E. Bompianr, Sulle connessioni affini non-posizionali, a Arch, d. Math. ", 3, 183-186, (1952).

[5] O. ONIcescu, Scostamento geodetico, stabilità e problema di Whittaker, *Rendic. Accad. Lincei s, $5,561.563,(1927)$.

[6] E. Bortolotri, Scostamento geodetico e sue generatizzazioni, "Giornale di Matematiche di Battaglini ", 64, 1.39, (1928).

[7] J. L. Synge, On the first and second variations of the line integral in Riemannian space, "Proe. London Math. Soc. ", 2, 25, 247-264, (1926),

[8] H. Rund, Zur Begründung der Differentialgeometrie in Minkonskischen Räumen. - Arch. d. Math. *, 3,60.69, (1952).

[9] H. Rund, The theory of subspaces of a Finsler space, Part. I, * Math. Z. „, 56, 363.37o, (1952).

[10] H. RUND, The theory of subspares of a Finsler space, Part. II, "Math. Z. ", 57, 193.210, (1952).

[11] W. BLASCuKe, Vorlesungen über Differentialgeometrie, Band I, Brd edition. Berlin, (1924).

[12] E. T. DAVIES, On the second variation of a simple integral with movable end-points, "Journ. London Math. Soc. ". 24, 241-247, (1949).

[13] C. CARATHÉODORX, Variationsrechnung und partielle Differentialgleichungen erster Ordnung, Berlin, (1985).

[14] G. A. Burss, Jacobi's Condition for problems in the Calculus of Variations in para. metric form. * Trans. Amer. Math. Soc. , 17, 195-206, (1916).

[15] O BouzA, Vorlesungen uber Variationsrechnung, Leipzig, (1909). 